\title{
Investigations the Electronic Structure in Monoclinic phase Gadolinium Sesquioxides by Electron Energy Loss Spectroscopy
}

\author{
SZ-CHIAN LIOU ${ }^{1}$, CHUN-HSIN KUO ${ }^{2}$ and GUO-JIAN SHU ${ }^{3}$ \\ ${ }^{1}$ UNIVERSITY OF MARYLAND, COLLEGE PARK, North Potomac, Maryland, United States, \\ ${ }^{2}$ TEXAS A\&M UNIVERSITY, United States, ${ }^{3}$ NATIONAL TAIPEI UNIVERSITY OF \\ TECHNOLOGY, United States
}

Gadolinium sesquioxide $\left(\mathrm{Gd}_{2} \mathrm{O}_{3}\right)$ has various applications in magnetic resonance imaging and optical coating material. Due to its high dielectric constant $(\kappa \sim 14)$, large band gap $(5.4 \mathrm{eV})$, and high refractive index of $1.8 \sim 2$, it has become a potential candidate material for metal-oxide semiconductor field-effect transistors (MOSFETs) [1]. It has been reported that two crystalline $\mathrm{Gd}_{2} \mathrm{O}_{3}$ phases, cubic phase (space group Ia3) and monoclinic phase (space group of $\mathrm{C} 2 / \mathrm{m}$ ), exist at ambient temperature and pressure [2], while cubic $\mathrm{Gd}_{2} \mathrm{O}_{3}$ has applied in most of the applications. In addition, monoclinic phase $\mathrm{Gd}_{2} \mathrm{O}_{3}$ (denoted as $\mathrm{m}-\mathrm{Gd}_{2} \mathrm{O}_{3}$ ) can usually obtain during the fabrication process [3]. Here, we study an electronic structure of $\mathrm{m}-\mathrm{Gd}_{2} \mathrm{O}_{3}$ performed by electron energy loss spectroscopy (EELS) in conjunction with scanning transmission electron microscopy (STEM) mode (STEM-EELS) and momentum (q)-dependence EELS (q-EELS).

TEM samples of $\mathrm{m}-\mathrm{Gd}_{2} \mathrm{O}_{3}$ powders, were synthesized through the process in ref. [3], were prepared by suspending $\mathrm{m}-\mathrm{Gd}_{2} \mathrm{O}_{3}$ powders in ethanol solvent, sonicating, and then drop onto a holey $\mathrm{C}$-coated $\mathrm{Cu}$ grid. STEM- and q-EELS were examined using a Thermo Fisher Themis 300 (scanning) transmission electron microscope [(S)TEM] equipped with electron monochromator and Gatan Image Filter (GIF, model Quantum 965), operating at $300 \mathrm{kV}$. The energy resolution with electron monochromator is $0.2 \mathrm{eV}$ throughout the STEM-EELS experiments. Real-space spectrum-imaging (SI) in energy-filtered TEM (EFTEM) mode with a tunable energy-selection slit was conducted on the same microscope. The single scattering EELS spectra were deconvoluted from raw data, and the subsequent Kramers-Krönig analysis (KKA) was conducted on the DigitalMicrograph EELS package, which is described elsewhere [4]. The qEELS experiments were carried out under diffraction mode with q resolution of $0.03 \AA-1$.

Figure 1(a) shows the STEM-EELS spectra acquired on $\mathrm{m}-\mathrm{Gd}_{2} \mathrm{O}_{3}$ with the incident electron probe positioned at various locations of the material as indicated in the HRTEM image [inset in Fig. 1(a)]. The blue curve in Fig. 1(a) reveals several spectral features at the energies of $\sim 7.5, \sim 10, \sim 15, \sim 17.8, \sim 27.5$, $\sim 31.5$, and $\sim 36 \mathrm{eV}$, respectively. Combining the $\varepsilon 1=0$ at $\sim 14.5 \mathrm{eV}$ in the frequency $(\omega)$-dependent dielectric function $[\varepsilon(\omega)=\varepsilon 1(\omega)+\mathrm{i} \varepsilon 2(\omega)]$ of $\mathrm{Gd}_{2} \mathrm{O}_{3}$ in Fig. 1(b) and the maximum intensity localized in the bulk interior in EFTEM-SI [see Fig. 1(e)], the spectral feature at $\sim 15 \mathrm{eV}$ can be interpreted as volume plasmons (VPs). Positioning the electron probe at the sample edge as indicated as a green circle and green curve in Fig. 1(a), the spectral intensity of the VP peak significantly decreases, and following up with visibly enhanced the peak at $\sim 13.6 \mathrm{eV}$. This result indicates the existence of surface-related excitation, surface plasmons (SPs), due to negative $\varepsilon 1$ in Fig. 1(b). This result is also consistent with the calculated relativistic E-k map (E, energy loss; $\mathrm{k}$, momentum transfer) for the 10 -nm thick $\mathrm{Gd}_{2} \mathrm{O}_{3}$ films inset in Fig. 1(b) exhibiting the peak maximum at $\sim 13.6 \mathrm{eV}$. By moving the incident electron probe further from the sample edge into vacuum [e.g., red and black curves in Fig. 1(a) with probe step of $2 \mathrm{~nm}$ ], the broad shoulder at $\sim 7$ and $\sim 9.5 \mathrm{eV}$ persists. Meanwhile, the spectral feature at $\sim 7 \mathrm{eV}$ bears a strong surface excitation character with evanescent wave field decaying into vacuum revealing by EFTEM-SI intensity map [Fig. 1(d)]. Combining with both STEM-EELS spectra and EFTEM-SI intensity maps, the spectral 
feature at $\sim 7 \mathrm{eV}$ peak can be considered as a surface resonance associated with excitonic onsets understood as surface exciton-polaritons (SEPs) [4]. In contrast to $\mathrm{VP}$ at $15 \mathrm{eV}$, the spectral intensity at $36 \mathrm{eV}$ peak decreases when position electron probe from the material interior to the specimen edge, and then vanished when the electron probe is positioned at $2 \mathrm{~nm}$ away from the material surface as shown in Fig. 1(a). Furthermore, the spectral feature at $36 \mathrm{eV}$ also exhibits maximum intensity localized in the bulk interior in EFTEM-SI [see Fig. 1(f)], which indicates the spectral feature at $36 \mathrm{eV}$ reveals a kind of volume excitations. Intriguingly, the q-EELS spectra in Fig 2(b) were obtained along the [001] direction in Fig. 2(a), reveal that the intensity of the spectral peak at $\sim 36 \mathrm{eV}$ increases with increasing the q values. Unlike the exponentially decaying in intensity as a function of q values for both SPs and VPs, the intensity of the spectral peak at $\sim 36 \mathrm{eV}$ increases as a function of $\mathrm{q}$ which implies the involving multiple scattering processes as described in ref. [4].

Research was supported by Maryland Nano Center at UMD, and MOST-Taiwan under project number of 108-2112-M-027-002-MY3.
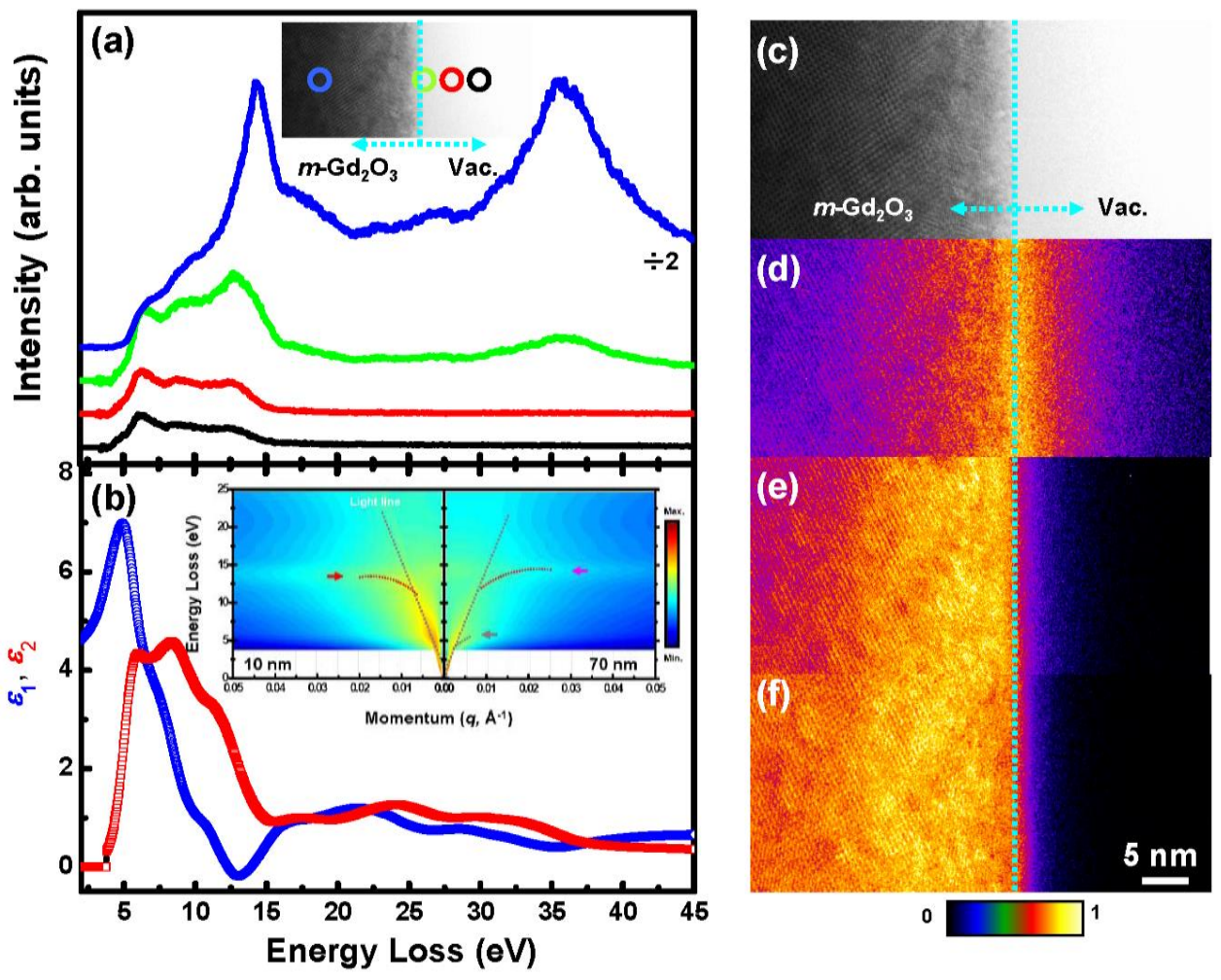

Figure 1. Fig. 1. (a) Position dependent EELS spectra acquired on $\mathrm{m}-\mathrm{Gd}_{2} \mathrm{O}_{3}$ with the electron probe positioned at various locations with color circles in inset HRTEM image. (b) The complex dielectric function of $\mathrm{m}-\mathrm{Gd}_{2} \mathrm{O}_{3}$ derived from the blue spectrum in (a). Inset is the calculated relativistic E-k maps ( $\mathrm{E}$ is energy loss and $\mathrm{k}$ is momentum transfer) for $\mathrm{Gd}_{2} \mathrm{O}_{3}$ slabs of a thickness of $10 \mathrm{~nm}$ (left) and $70 \mathrm{~nm}$ (right panel). (c-f) The EFTEM-SI intensity maps acquired from the HRTEM image (c) at different energies of $6 \sim 8 \mathrm{eV}(\mathrm{d}), 14 \sim 16 \mathrm{eV}(\mathrm{e})$, and $35 \sim 37 \mathrm{eV}(\mathrm{f})$. The dotted blue lines indicate the interface 
between material (left part) and vacuum (right part). The color scale bar represents the linearly normalized image intensity.
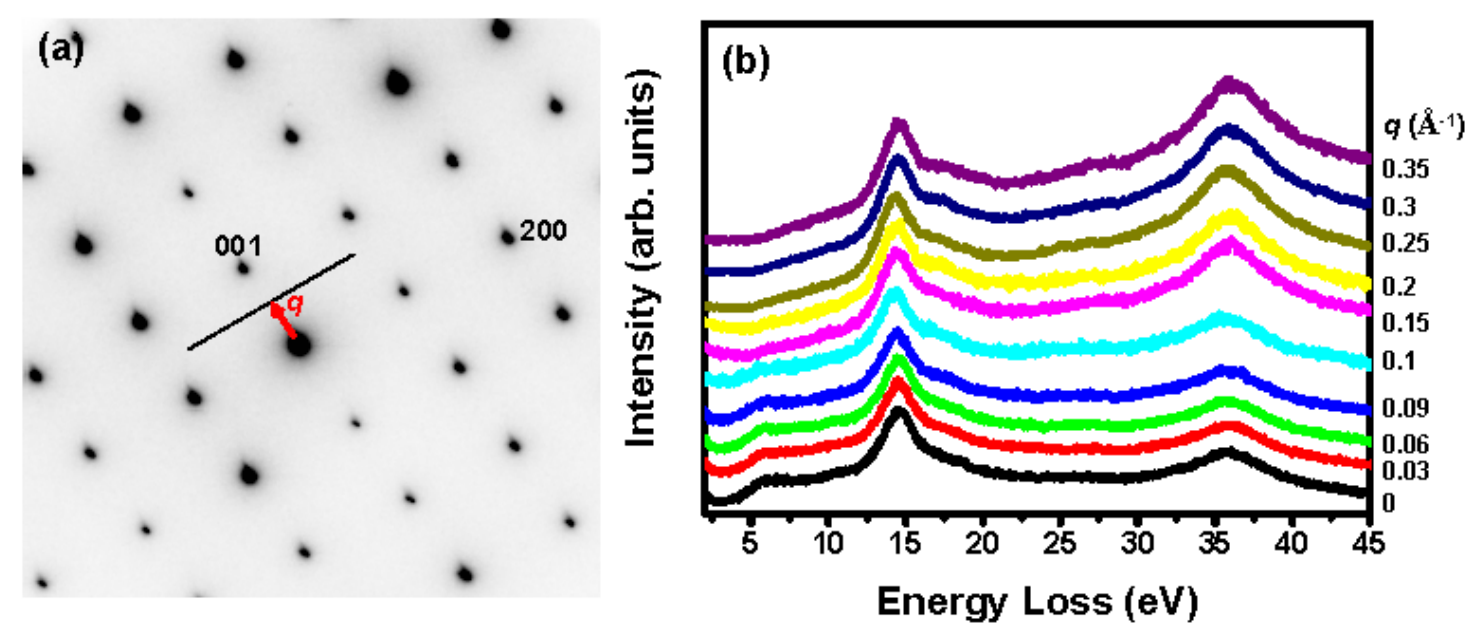

Figure 2. Fig. 2. (a) Electron diffraction pattern acquired along [010] incident electron beam direction. The black-color dash line in (a) indicates the Brillouin zone (B. Z.). The red arrow in (a) shows the momentum transfer range for q-EELS experiments. (b) The q-EELS spectra obtained along the $\mathrm{q}$ direction along [001] directions in (a).

\section{References}

[1] M. Hong, J. Kwo, A. R. Kortan, J. P. Mannaerts, and A. M. Sergent, Science 283, 1897 (1999).

[2] F. X. Zhang, M. Lang, J. W. Wang, U. Becker, R. C. Ewing, Phys. Rev. B 78, 064114 (2008).

[3] L. Sun, C. Liao, and C. Yan, J. Solid State Chem. 171, 304 (2003).

[4] G. J. Shu, S. C. Liou, et al., AIP Advances 10, 105022 (2020). 